Check for updates

Cite this: RSC Adv., 2019, 9, 34472

Received 22nd July 2019

Accepted 3rd October 2019

DOI: 10.1039/c9ra05645a

rsc.li/rsc-advances

\title{
Biomaterial-based microstructures fabricated by two-photon polymerization microfabrication technology
}

\author{
Xiaoying Wang, ${ }^{a}$ Zhenping Wei, ${ }^{a}$ Charles Zuwu Baysah, ${ }^{a}$ Meiling Zheng (DD *b \\ and Jinfeng Xing (iD *a
}

Two-photon polymerization (TPP) microfabrication technology can freely prepare micro/nano structures with different morphologies and high accuracy for micro/nanophotonics, micro-electromechanical systems, microfluidics, tissue engineering and drug delivery. With the broad application of 3D microstructures in the biomedical field, people have paid more attention to the physicochemical properties of the corresponding materials such as biocompatibility, biodegradability, stimuli responsiveness and immunogenicity. Therefore, microstructures composed of biocompatible synthetic polymers, polysaccharides, proteins and their complexes have been widely studied. In this review, we briefly summarize the TPP mechanism, the photoinitiators for TPP microfabrication, photoresist based on biomaterials, their corresponding microstructures and subsequently their biomedical applications. We will point out the issues in previous research and provide a useful perspective on the future development of TPP microfabrication technology.

\section{Introduction}

The rapid development of micro-manufacturing technologies enables us to prepare micro/nano structures accurately for medical devices, ${ }^{1-3}$ biosensors ${ }^{4}$ and optical devices. ${ }^{5-10}$ Many micro/nano manufacturing technologies have been extensively developed and reported based on the characteristics of the materials and fabrication methods, including electron beam lithography, ${ }^{\mathbf{1 1}, 12}$ ion beam processing technology, ${ }^{\mathbf{1 3 , 1 4}}$ laserbased rapid printing $(\mathrm{RP})^{15}$ and nanoimprint technology. Laser-based RP technology has developed rapidly in recent years, including selective laser sintering (SLS), stereolithography (SLA), ${ }^{16}$ micro-stereolithography ( $\mu$-SLA),${ }^{17}$ solid ground curing (SGC) ${ }^{18,19}$ and two-photon polymerization (TPP) microfabrication technology. Among them, TPP microfabrication technology can freely prepare micro/nano structures with different morphologies and high accuracy for micro/ nanophotonics, ${ }^{7}$ micromachine systems, ${ }^{\mathbf{2 0}}$ microfluidics, ${ }^{21}$ tissue engineering and drug delivery. ${ }^{22}$ Fig. 1 summarizes the different laser-based RP techniques and simply distinguishes the light sources and materials used in laser-based RP techniques.

${ }^{a}$ School of Chemical Engineering and Technology, Tianjin University, Tianjin, 300350, China.E-mail:jinfengxing@tju.edu.cn

${ }^{b}$ Laboratory of Organic NanoPhotonics and CAS Key Laboratory of Bio-Inspired Materials and Interfacial Science, Technical Institute of Physics and Chemistry, Chinese Academy of Sciences, No. 29, Zhongguancun East Road, Beijing, 100190, P. R. China. E-mail: zhengmeiling@mail.ipc.ac.cn
Compared to single-photon photopolymerization, TPP only occurs in the laser focal area with a threshold up to enough photon density. Therefore, microstructures with high accuracy beyond the diffraction limit can be fabricated. TPP microfabrication as a kind of femtosecond laser direct writing technology can selectively photo-cure sticky liquids to obtain threedimensional (3D) microstructures with pre-designed morphologies. ${ }^{22}$ Transparent liquid reduces the attenuation rate of laser light and transforms into polymer with a high glass transition

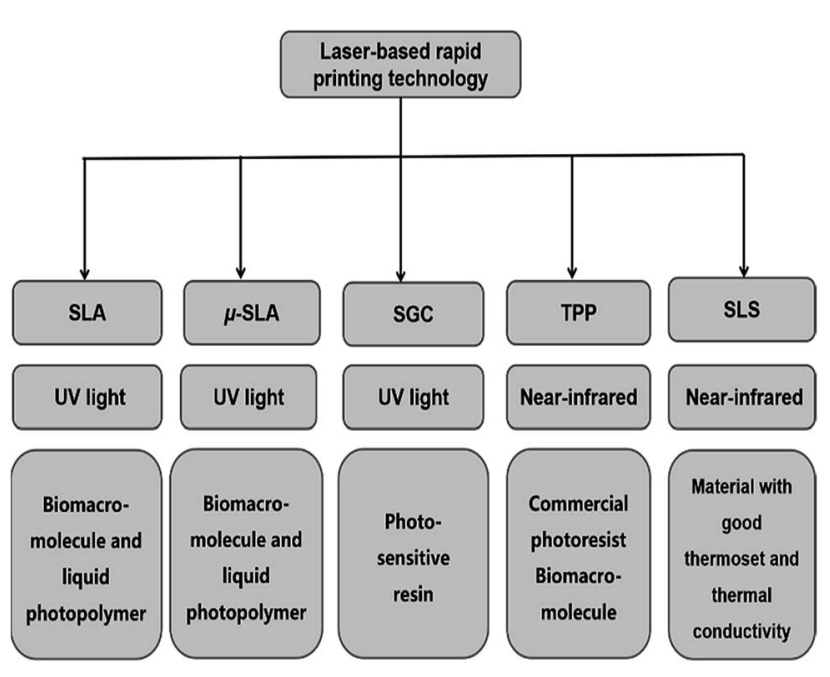

Fig. 1 Classification of laser-based rapid printing technologies. 
temperature after exposure. Commercial photoresists such as SU-8, ${ }^{21,23-25}$ SCR500, ${ }^{26,27}$ SZ2080 (ref. 28-30) and NOA61 (ref. 31) have been widely used in TPP microfabrication. Acrylate-based polymers show biocompatibility at some extent. However, their hydrolysis products are harmful. Therefore, vinyl estermodified monomers have been used for TPP and their corresponding degradation products are less cytotoxic compared to acrylates. $^{32}$

With the broad application of 3D microstructures in the biomedical field, people have paid more attention to the physical and chemical properties of polymeric materials such as biocompatibility, biodegradability, stimuli responsiveness and low immunogenicity. Therefore, microstructures composed of polysaccharides, ${ }^{33}$ proteins $^{34}$ and their complexes ${ }^{35}$ have been widely studied. As a typical polysaccharide, hyaluronic acid widely distributed in human tissues is a linear polysaccharide in the extracellular matrix. ${ }^{36}$ As a natural degradable polymer, it has been extensively used in the biomedical field. ${ }^{37,38}$ Proteins are composed of a variety of amino acids with many modified amino and carboxyl groups. Proteins including elastin, silk protein and collagen have been extensively studied. ${ }^{39}$ Sun et al. used regenerative silk protein to prepare microstructures with different shapes, and controlled processing parameters and doped metal gold to obtain microelectrodes with adjustable conductivity via two-photon photo-crosslinking. ${ }^{40}$ Gelatin is a biomaterial produced by hydrolysis of collagen and often used in foods and chemicals. Gelatin can be modified with methacrylamide, vinyl ester and acrylamide to prepare a micro structure for cell culture and drug delivery. ${ }^{\mathbf{4 1 - 4 4}}$ Although a large number of biomaterials-based microstructures prepared with TPP microfabrication technology have been reported, ${ }^{45-47}$ few reviews about the preparation methods, process parameters and their applications have been reported.

This paper summarizes the development of TPP microfabrication technology for the fabrication of microstructures based on biomaterials, including a brief review of TPP mechanism, photoinitiators, photoresist based on biomaterials, their corresponding microstructures and subsequently their biomedical applications.

\section{Mechanism and experimental setup for TPP}

As a rapid prototyping technology, TPP microfabrication uses near-infrared laser with deep penetration as the light source. The components of photoresists participating in TPP contain monomer, cross-linker and photoinitiator. The transparent negative photoresist with small Rayleigh scattering constant reduces the absorption loss of laser. Commercially available photoresists, polysaccharides (HA and its derivatives), ${ }^{33}$ PEGbased $^{\mathbf{4 8}}$ and protein-based ${ }^{\mathbf{4 9}}$ photoresists undergo highly localized chemical reactions in the presence of photoinitiator. The photoinitiator simultaneously absorbs two photons under the laser radiation which leads to an excited state, and then initiates a crosslinking or stepwise growth reaction of the monomers.

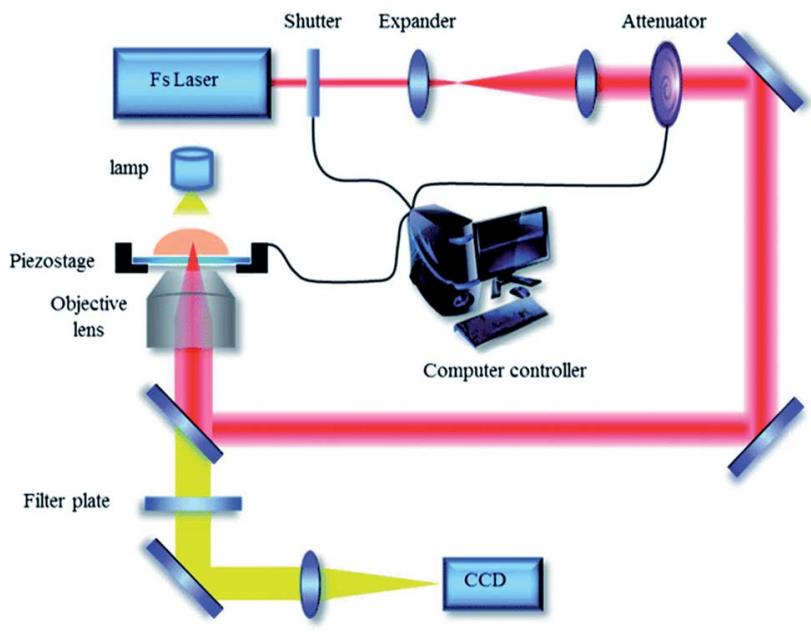

Fig. 2 Experimental setup for TPP microfabrication in our lab (reprinted from ref. 50 with permission from Royal Society of Chemistry).

The process of TPP microfabrication is illustrated by using the experimental setup in our lab (Fig. 2). ${ }^{50} \mathrm{~A}$ near-infrared femtosecond laser is used as a laser source with a specific pulse width and frequency. The oil immersion objective lens equipped on the device is introduced to focus the beam.

Different microstructures are fabricated after the focused beam irradiate on the photoresist loaded on an XYZ stage controlled with computer software. The uncured photoresist is removed by ethanol after fabrication. Due to the difference of the laser energy density distribution, high photon density at focus center of the laser beam provides greater possibility of two-photon absorption, which results in the threshold for TPP, and TPP can be confined in the region near the focus center. Therefore, the spatial resolution of TPP can be precisely tuned by optimizing laser processing parameters such as laser power, exposure time, initiator concentration and free radical quenchers to control the polymerization region. ${ }^{51-53}$ The micro/ nano lines, ${ }^{51}$ micropump, ${ }^{54}$ micro-needles, ${ }^{55}$ cantilever beams, ${ }^{56}$ micro-array ${ }^{57}$ and other micro-structures ${ }^{51}$ have been prepared under the control of the computer according to the layer-bylayer bottom-up processing method. Compared to conventional micro-manufacturing techniques, TPP microfabrication as an effective laser direct writing technology exhibits excellent spatial resolution. ${ }^{22}$ The factors influencing the processing accuracy has been discussed in detail. ${ }^{58}$

\section{TPP photoinitiator}

Photoinitiator, as an important component in TPP, absorbs two photons at long wavelength with low energy to convert from the ground state to the excited state, which then initiates the monomer to generate radicals. Photoinitiators include hydrophilic and hydrophobic ones (see Table 1). Hydrophobic photoinitiators generally have large two-photon absorption crosssection $(\delta)$ which is beneficial to the initiation of the polymerization. According to the symmetric charge transfer, the bis- 
Table 1 Photoinitiators used in two-photon polymerization

\begin{tabular}{|c|c|c|c|c|c|}
\hline PIs & Design strategies & Core structures & $\lambda_{\max }(\mathrm{nm})$ & $\sigma^{2 \mathrm{PA}}(\mathrm{GM})$ & Ref. \\
\hline \multirow[t]{2}{*}{ Water-insoluble TPIs } & \multirow[t]{2}{*}{ Symmetric charge transfer } & Bis(styryl) benzene & 810 & 3670 & 59 \\
\hline & & Bis(styryl) benzene & 775 & 1250 & 60 \\
\hline \multirow[t]{3}{*}{ Water-soluble TPIs } & \multirow[t]{3}{*}{ Supramolecular self-assembly } & Anthraquinone, cyclodextrins & 780 & 200 & 50 \\
\hline & & Anthraquinone, PF127 & 780 & 200 & 63 \\
\hline & & BMVPC-CB7 & 567 & 2999 & 64 \\
\hline & Xanthene & Erythrosin & 800 & 10 & \\
\hline & \multirow[t]{3}{*}{ Benzylidene cyclanone } & $\mathrm{T} 1$ & 820 & 567 & 71 \\
\hline & & $\mathrm{T} 2$ & & 808 & \\
\hline & & $\mathrm{T} 3$ & & 231 & \\
\hline
\end{tabular}

(styryl)benzene derivatives with large $\delta$ were synthesized. The $\delta$ was increased by charge redistribution on donor and acceptor groups on bis-(styryl)benzene derivatives. $\delta$ of the molecules exhibited 400 times than that of trans-stilbene. ${ }^{59}$ Series of $\pi$ conjugated derivatives were developed by Perry et al. They showed large $\delta$ due to their strong two-photon absorption capacity. ${ }^{60} \quad C_{2 \mathrm{v}}$ symmetrical 2,7-bis[2-(4-substituted phenyl)vinyl]-9,10-dipentyloxyanthracenes were synthesized by Xing et al. They possessed low thresholds and high photoinitiating sensitivity due to special symmetrical structure and strong electron donating ability. ${ }^{61}$

Although hydrophobic initiators display large $\delta$, the use of them in TPP microfabrication for biomedical application is restricted. Hydrophobic initiator can be changed into its watersoluble counterpart by introducing a nonionic surfactant or modifying a hydrophilic group on it. Ober et al. firstly increased the solubility of 2,2-dimethoxy-2-phenyl acetophenone (Irgacure 651) in water using the nonionic surfactant of Pluronic F127 (PF127). 3D hydrogel microstructures were prepared by initiating polymerization of 2-hydroxyethyl methacrylate (HEMA) and poly(ethylene glycol) diacrylate (PEGDA). ${ }^{62}$ Xing et al. combined PF127 and 2,7-bis(2-(4-pentaneoxy-phenyl)vinyl)anthraquinone through hydrophobic interaction to prepare a water-soluble initiator with high initiating efficiency, which initiated polymerization of PEGDA to prepare hydrogel lines with a width of $92 \mathrm{~nm}$. Furthermore, the adenoviruses microstructure with high precision was fabricated. ${ }^{63}$

Besides hydrophobic interaction, host-guest interaction is also used to prepare water-soluble photoinitiators. Xing et al. prepared a water-soluble initiator with high initiating efficiency by utilizing the host-guest interaction. This watersoluble initiator showed high $\delta$ and low threshold power (Fig. 3). ${ }^{50}$ Duan et al. prepared water-soluble BMVPC-CB7 with five-fold $\delta$ than that of BMVPC before interaction with CB7. The BMVPC-CB7 was synthesized based on the hostguest interaction between carbazole vinylpyridinium derivative and cucurbit[7] uril (CB7). The CB7 complex initiator was used to induce PEGDA to prepare hydrogel scaffolds with a resolution up to $180 \mathrm{~nm}$ under a low power threshold of 4.5 mW. ${ }^{64}$ Besides the physicochemical properties of the initiators, the microenvironment of the reaction should be considered to maximize the initiator's ability to produce the radicals. Most of the water-soluble initiators that initiate protein cross-linking are xanthene dyes, including methylene blue, rose bengal, and so on. ${ }^{49,65}$ The initiating efficiency of rose bengal is sensitive to the $\mathrm{pH}$ value. Under acidic conditions, the activity of chromophore in the rose bengal disappears. To solve this problem, Campagnola et al. modified the benzophenone dimer (BPD). The initiator showed solubility in the protein matrix and was retained in the protein microstructures after photocrosslinking. The final structure not only had excellent cell adhesion but also showed good biological activity. The degradation rate of the proteins can be regulated by tuning the crosslink density. ${ }^{\mathbf{6 6}}$

Compared with oil-soluble photoinitiators, water-soluble photoinitiators are more attractive by virtue of initiating the photocrosslinking of proteins in an aqueous environment without introducing organic toxic substances. A series of xanthene dyes including rose bengal, eosin Y, erythrosin, fluorescence, rhodamine $\mathrm{B}$, and coumarin 519 have been used as photosensitizers in TPP. $^{67}$ Researchers have explored and compared their initiation efficiency, and the features of

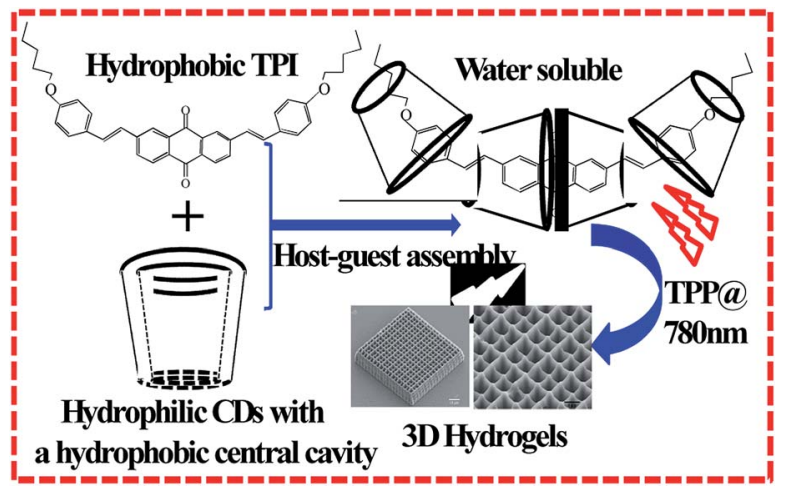

Fig. 3 Water-soluble TPI synthesized by host-guest interaction between hydrophobic TPI and hydrophilic CDs and used in 3D hydrogel microstructure fabrication (reprinted from ref. 50 with permission from Royal Society of Chemistry). 
synthetic 3D structures were characterized. Photosensitizers and photoinitiators have two different mechanisms of action as photoreceptors. $^{68}$ Photoinitiators are classified into the hydrogen scavenging photoinitiator, and the co-initiator which acts as a hydrogen donor. Under illumination, the initiator captures the hydrogen atoms of the co-initiator to form free radicals to initiate polymerization. The other initiator absorbs enough energy under laser radiation to break itself to form free radicals. ${ }^{69}$

Campagnola et al. proposed a novel idea to combine the functions of photosensitizers and co-initiators in the same polyfunctional molecules and successfully synthesized rose bengal diisopropyl amine derivative and BPD photoinitiator with flexible diamine tether. The initiator was successfully used to cross-link bovine serum albumin (BSA) and collagen. Compared with traditional benzophenone, the initiating efficiency was increased obviously and the formed proteins was structurally stable and highly modifiable. ${ }^{70}$ The efficiency of TPP is largely determined by the $\delta$ of the photoinitiators. The benzylidene cyclanone dyes with large $\delta$ and excellent biocompatibility have been used to prepare $2 \mathrm{D}$ and $3 \mathrm{D}$ microstructures of protein, acrylates (SR610) and modified HA. The maximum of $\delta$ reaches $808 \mathrm{GM}$, which greatly reduces the laser energy required to initiate polymerization, and the outstanding biocompatibility shows its advantage in biomedical application. ${ }^{71}$ In recent years, although many studies have been done on the water solubility, toxicity and $\delta$, the preparation of water-soluble photoinitiators with high initiating efficiency and biocompatibility should be further explored.

\section{Photoresists for TPP and their corresponding microstructures}

\subsection{Photoresists based on synthetic molecules and their corresponding microstructures}

The photoresists for TPP are viscous liquids, which are solidified after irradiation of the laser to produce pre-designed microstructures. The commercialized photoresists such as acrylate SCR500, epoxy-based SU-8, SZ2080 and NOA61 have been widely used to prepare microstructures. ${ }^{23-31}$

In addition to commercial photoresists, pentaerythritol triacrylate (PETIA) and PEGDA have also been polymerized into various microstructures (see Table 2). Farsari et al. chose PETIA as transparent monomer and eosin $\mathrm{Y}$ as initiator to fabricate the gear transport equipment used as photonic devices, actuators and micro-fluidic devices. ${ }^{72}$ As food additives, pharmaceutical intermediates and raw materials for cosmetic and other industries, PEG belongs to biocompatible material. Therefore, PEGDA has been widely used to prepare microstructures for biomedical application.

PEGDA has been prepared into microstructures of various sizes and arbitrary geometries by TPP microfabrication technology. Cells adhering to the microstructure retain cell activity and proliferation. These microstructures can be used in tissue engineering to achieve multicellular tissue constructs.
Avsianikov et al. combined TPP microfabrication technology with laser-induced transfer technology to prepare PEG cell culture medium with high-porosity in vitro. The pore sizes in this culture substrate not only facilitate the transport of nutrients and metabolic wastes but also promote cell attachment, division and migration. ${ }^{73}$ Liefeith et al. explored PEGDA to fabricate biological scaffolds and studied the biological activity of the cultured cells. The size of the scaffold was amplified to increase the practical possibilities of extracellular matrix to the cell culture. ${ }^{74}$

The mechanical property of the microstructure is also an important factor influencing cell viability. Xing et al. explored the relationship between Young's modulus of PEGDA microstructures and processing parameters such as laser power, exposure time and $Z$-axis distance. Furthermore, microstructures like the form of red blood cell were fabricated at arbitrary angle, which has potential application in tissue engineering and drug delivery. ${ }^{48}$ Besides PEGDA with low molecular weight is used to prepare substrates, supports and microarrays with distinct pore sizes and morphologies, PEGDA with high molecular weight is also used in similar fields. Scarpa et al. used PEGDA with high molecular weight $(10 \mathrm{kDa})$ to prepare micropatterns such as pyramids and domes. Compared to polymers composed of PEGDA with low molecular weight, polymers composed of PEGDA with high molecular weight exhibit more excellent mechanical properties. ${ }^{75}$ Survival and differentiation behaviors of cells on the substrate are affected by the types of photosensitive resins, photoinitiators and the residual monomers. Ovsianikov et al. explored the cell viability ratio of various PEG derivatives on different substrates initiated by versatile photoinitiators such as Irgacure 369 and Irgacure 2959, and further optimized the biocompatibility of the substrates. ${ }^{76}$

PEGDA-based microneedles have also been used in biological drug delivery systems. However, epidermal bacterial infections caused by microneedles should be considered. Gentamicin sulfate and PEGDA were cross-linked to prepare a uniform microneedle array. The growth of bacteria on the surface of the microneedle array was suppressed by gentamicin sulfate, and the risk of bacterial infection was greatly reduced during medical administration. ${ }^{77}$

The drug carriers with environmental responsiveness such as $\mathrm{pH}$, temperature, and humidity can effectively increase the targeted release of drug and reduce the drug dose and side effects on the human body. For example, the humidity responsiveness of PEGDA substrate was explored by Sun et al. ${ }^{78}$ Reversible humidity-responsive hydrogel grids with cyclic stability simulating the movement of the pores in the plant were prepared, and the corresponding brakes and sensors with humidity stimulation response function were prepared. In order to obtain more stimuli responsiveness, the physicochemical properties of the fabricated microstructures have been modified by doping polymers with carboxylic acid groups, metals and inorganic materials. PEGDA microstructures that impart environmental responsiveness can also be used as sensors, varyers and micro-robots. 
Table 2 Materials explored in two-photon polymerization

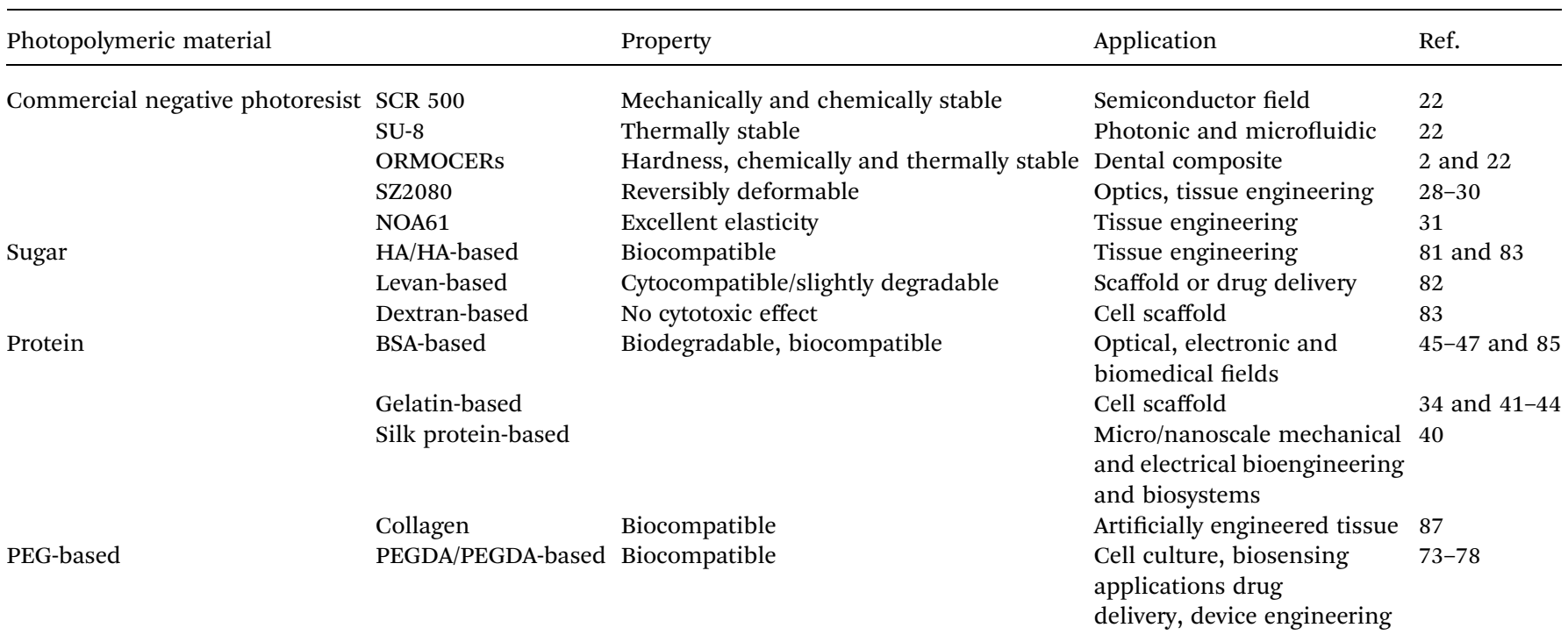

\subsection{Photoresists based on biomacromolecules and their corresponding microstructures}

Photosensitive resins composed of synthetic polymers have attractive chemical flexibility and can form functional microstructures through the introduction of special functional groups. However, the biodegradability and biocompatibility as well as immunogenicity of the microstructures can not meet the requirement in biomedical application. ${ }^{79}$ Unlike synthetic polymers, biomacromolecules including proteins, sugars and so on have excellent biodegradability, biocompatibility and low immunogenicity.

4.2.1 Photoresists based on polysaccharide and their corresponding microstructures. Polysaccharides are natural macromolecular materials composed of monosaccharides and have outstanding degradability. They are often used to prepare hydrogel with TPP. As a typical polysaccharide, HA can facilitate cell differentiation and form hydrogel framework with excellent physicochemical properties through TPP microfabrication technology. ${ }^{80}$ Kufelt et al. considered that the mechanical properties of the individual HA hydrogels could not meet the practical requirements. Therefore, HA and PEGDA were combined to form the microstructures. Epidermal growth factor (EGF) with the function to strengthen cell viability was introduced into the HA structure to enhance cell survival on the modified HA substrate. ${ }^{\mathbf{8 1}}$ In addition, a series of polysaccharides have been modified with photocurable chemical groups, such as dextran methacrylate, hyaluronic acid methacrylate, polyglycerol carbamate ester methacrylate, ${ }^{82}$ Levan urethane methacrylate, Levan methacrylate and so on. ${ }^{83}$

4.2.2 Photoresists based on protein and their corresponding microstructures. Proteins composed of amino acids possess enzymatic degradability, molecular composition flexibility and chemical modification ability. Therefore, proteins have the potential to substitute for synthetic polymers. Proteins and their derivatives exhibit a series of excellent physicochemical properties such as satisfactory elasticity, sufficient mechanical strength and electromagnetic property. ${ }^{\mathbf{4 1 , 8 4}}$ Protein microfilaments, arbitrary micropatterns, 3D non-adhering structures, microlenses and the likes have been prepared by TPP microfabrication. ${ }^{\mathbf{4 0 , 4 5 , 8 5}}$

BSA is globulin isolated from bovine serum and consists of amino acids with many oxidizable residues. The photocrosslinking reaction of BSA is performed by absorption of groups such as tryptophan or carried out by the peroxy radicals generated by the photosensitive material oxidizing the amino acid residues. ${ }^{49}$ BSA has been used in TPP microfabrication to produce non-toxic micro/nano devices used for biomimetic engineering and therapeutic delivery. Cellular activity is affected by the characteristics of the microstructure such as elasticity and stiffness. The elastic modulus of the microcolumns and the number of pores as well as pore size of the surface micropores decrease with the laser power increasing. As the concentration of the reaction solvent increases, the pore diameter and porosity decrease. The stiffness of the microcolumns is affected by the height. For cytology, the interplay between different types of cells and microcolumns increases as the stiffness of the microcolumns strengthens. ${ }^{86}$

There is still a challenge that the stiffness of the protein matrixs and cell viability are simultaneously improved. Kuetemeyer et al. presented a method to produce highly rigid artificial collagen-based tissue constructs in the presence of riboflavin. The rigidity of the matrix was increased about $40 \%$ and the activity and function of cells were not affected. ${ }^{87}$ Cell proliferation and differentiation behaviors are precisely controlled by TPP microfabrication. Shear et al. showed that protein matrixes can be functionalized either through direct cross-linking of enzymes such as biotinylated enzymes or by cross-linking biotinylated proteins that were then linked to biotinylated enzymes via an avidin couple. Several functional protein microstructures were prepared, including microparticles that could be 
translocated to desired sites of action including cytosolic positions, protein pads that generated product gradients within cell cultures and oncolumn nanoreactors for microfluidic systems. ${ }^{\mathbf{8 8}}$

Desired tissue structures depend on the directional migration ability of cells. Shear et al. developed a powerful method to pattern biotinylated and cell-adhesive cues on micro-3D-printed protein matrixes following two-photon-promoted conjugation of biotinylated benzophenone to scaffolds (via a NeutrAvidins bridge). Two-photon excited activation of benzophenone promotes biotin conjugation to scaffolds at desired positions with micrometer-scale. This strategy provides a means to tailor scaffold surface chemistry in three dimensions to elucidate cell matrix interactions and direct cell behavior. ${ }^{89}$ Shear et al. used two-photon excitation to photocrosslink protein microstructures within three-dimensional and optically transparent hydrogel materials based on HA. This unique method can be used to create complex three-dimensional architectures that provide both chemical and topographical cues for cell culture and guidance, providing for the first time a method to direct cell adhesion and migration on size scales relevant to in vivo environments. Furthermore, guidance of both dorsal root ganglion cells (DRGs) and hippocampal neural progenitor cells (NPCs) along arbitrary and three-dimensional paths was demonstrated. ${ }^{\mathbf{8 4}}$

BSA-based microstructures can be used as drug delivery vehicles. BSA, fibrinogen, fibronectin and globulin were photocrosslinked, and the first two proteins proved the retention of bioactivity after cross-linking. The pore size of the cross-linked matrixes was controlled by the crosslink density that affected the migration of drugs or active substances in the matrixes. ${ }^{\mathbf{9 0}, \mathbf{9 1}}$ Campagnola et al. studied the photochemical reaction efficiency of BSA and fibrinogen, and the application of fabricated matrixes as the drug carriers. ${ }^{91}$ The intelligent responsiveness of sustained drug delivery carriers is important. Xing et al. reported the BSA microstructures with dynamic response to $\mathrm{pH}$. The parameters were optimized to prepare BSA microstructures with a wide range of $\mathrm{pH}$ response. A panda facial micropattern with unique facial expression changes and mesh microsieve structures with changing pore sizes in different $\mathrm{pH}$ values were fabricated (Fig. 4). ${ }^{49}$

BSA-based microstructures have been used in the field of optics and materials science. The preparation of 3D complex independent microstructures promotes the application of two-

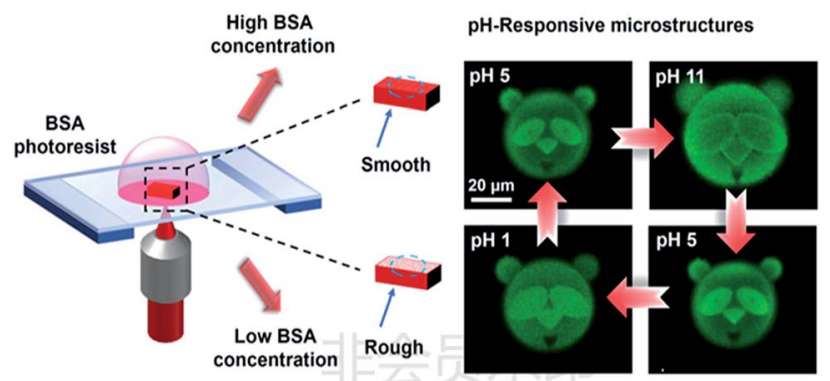

Fig. $4 \mathrm{pH}$-responsive panda facial micropattern (reprinted from ref. 49 with permission from American Chemical Society). photon microfabrication technology in micro-brakes and microfluidics. Sun et al. used the $\mathrm{pH}$ responsiveness of BSA to prepare protein microlens with reversible focal length ${ }^{\mathbf{8 5}}$ and tunable microwave diffraction microlens. The optical components are transformable and non-toxic. Compared with organic materials, protein microlens belong to an environmentally friendly micro-optical device. ${ }^{92}$ Microstructures fabricated by two-photon microfabrication technology shows special architecture. Shear et al. innovated a method to evaporate the original gel mixtures to obtain protein-based resist with high concentration and viscosity, which can overcome the problem of object movement during the preparation process. Complex 3D microstructures that are not adhered to a surface, including chains of Möbius strips, paddlewheels and unconstrained (freefloating) probes for bacterial motility were fabricated, which broke through the integrated structure formats between traditional microstructures and the preparation substrate surfaces. This provided the flexible mobility and free rotation for the resulting structures. ${ }^{45}$ Takeuchi et al. used flavin adenine dinucleotide to initiate BSA cross-linking, and prepared protein microwires, spiral structures and polyhedral frameworks completely out of plane (Fig. 5). The resolution of these structures depended on the preparation parameters. ${ }^{46}$

The fabrication parameters strongly influence the physicochemical properties of the microstructures. The rigid materials or the micro-mechanical materials with desired properties have been developed by selecting the process parameters. Fabrication parameters can be carefully tuned to prepare BSA microstructures with special mechanical properties. The micropillar array with uniform stimuli responsiveness was fabricated by adding chemically inert materials into the photoresist to adjust the viscosity. ${ }^{47}$ Photocrosslinking of most proteins are induced by RB. The excitation fluorescence signals of BSA microstructures prepared by RB inducing two-photon photocrosslinking

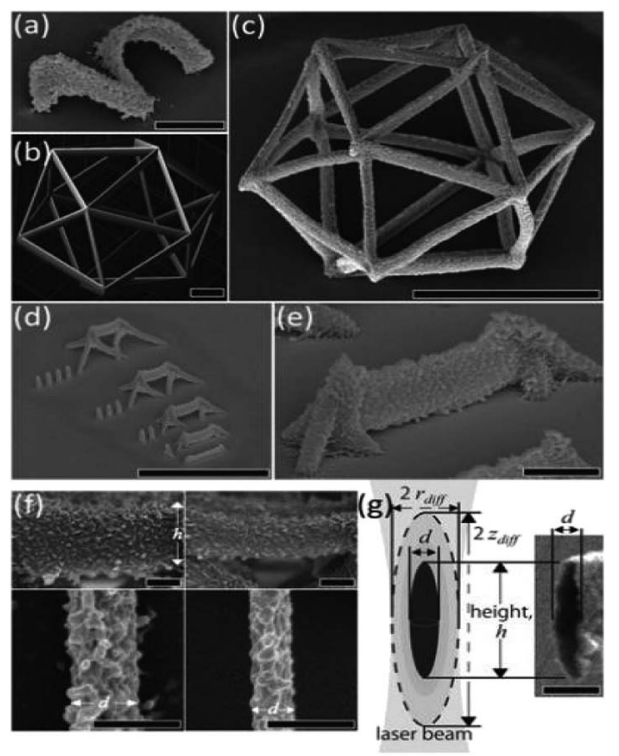

Fig. 5 SEM images of unattached BSA independent microstructure (reprinted from ref. 46 with permission from AIP Publishing). 
are greatly enhanced. The cross-linking degree of proteins can be monitored by the changes in fluorescence of microstructures originated from RB. ${ }^{93}$

Microstructures with environmentally responsive ability have been used both in drug delivery and micro-braking applications such as micro-robots, traps and so on. The high spatial resolution allows two-photon microfabrication technology to accurately and successfully prepare protein microstructures with nano-scale stiffness. By changing the $Z$-axis parameters in the operation, the cross-linking density of BSA can be adjusted on the nanoscale to achieve a programmed angular bending and orientation. The composite materials with quantitative dynamic response can be prepared as microcapture device. ${ }^{94}$ Furthermore, the functionalized proteins have been developed by the photosensitizers and reagents with special functional groups directly reacting with the monomeric proteins, or by doping other biological macromolecules or active substances. Shear et al. combined BSA, avidin and lysozyme to prepare arched and rectangular microstructures, and varied the component contents to obtain chemically responsive micromechanical components with different swelling behaviors. Microstructures as bacterial cages were fabricated with predictable directional bending behavior, which had the function to collect cells by controlling the differences in microlayer thickness and crosslink density. ${ }^{95}$

As an important component of the extracellular matrix, collagen can produce gelatin as an environmentally friendly hydrogel material which owns low immunogenicity, high biocompatibility, solubility and enzymatic hydrolysis. The microstructures with cell culture capacity have been prepared by chemical modification of gelatin using functional groups such as methacrylamide and methacrylate. ${ }^{\mathbf{4 1 - 4 4}}$ 3D scaffolds for tissue engineering were developed from methacrylamide-modified gelatin using TPP with the biocompatible Irgacure 2959 as the photoinitiator. The scaffolds were suitable to support porcine mesenchymal stem cell adhesion and subsequent proliferation. The gelatin derivative preserved its enzymatic degradation capability after photopolymerization. The developed scaffolds supported primary adipose-derived stem cell (ASC) adhesion, proliferation and differentiation into the anticipated lineage. ${ }^{\mathbf{4 1 4 2}}$ The physicochemical properties, biological properties and crosslink density of the gelatin derivatives are related to the number of photoactivatable groups.

Vlierberghe et al. synthesized a novel gelatin-based derivative for TPP. After the second modification of GelMOD, a gel-MODAEMA hydrogel material with nearly $100 \%$ biocompatibility was obtained, and the number of photocrosslinking reaction groups increased per unit time, which improved the reaction efficiency. The crosslinking density of the structure increased with adjustable mechanical properties and reduced the deformation problems of GelMOD hydrogel. ${ }^{96}$ Brusatin et al. confirmed that the non-toxic wood-like GELMA hydrogel microstructures with high resolution allowed cell immersion. The spacing of the wood-like structures and the rigidity of the structure were affected by laser power and exposure time. ${ }^{43}$

\section{Conclusions}

Various 2D and 3D micro/nano structures with high accuracy have been prepared by TPP microfabrication technology. The microstructures have potential applications in optical component manufacturing, MEMS, tissue engineering, and drug delivery. Before TPP microfabrication technology acquires real applications, some issues should be solved.

Photoinitiators play a key role in the absorption of light energy to initiate two-photon polymerization. Three strategies for designing photoinitiators should be considered. Firstly, more attention should be paid to design and synthesis of symmetric two-photon initiators to increase the two-photon absorption cross section. Secondly, water solubility is necessary since the synthetic or natural materials are photocrosslinked in aqueous environment. However, most photoinitiators with large absorption cross section are not only insoluble in water but also do not mix well with photoresist. Several strategies can be used to improve the water solubility of photoinitiators such as hydrophilic hydrophobic assembly, host-guest interaction and chemical modification. Finally, the toxicity of initiators should be as low as possible. Many methods can be used to reduce the toxicity of initiators including application of biocompatible initiators and decrease of initiator amount by increasing their initiating efficiency.

Biocompatible and degradable materials such as HA, gelatin and protein have been widely used in TPP microfabrication technology to prepare various microstructures for biomedical applications. However, the mechanical properties and topography of microstructures limit their applications. To solve those issues, the mixed photoresist can be designed to prepare microstructures by doping antimicrobial materials, growth factors, organic polymers and modified ceramics. For instance, the introduction of antimicrobial materials into photoresist can reduce the risk of infection caused by microneedle delivery drugs.

\section{Conflicts of interest}

There are no conflicts to declare.

\section{Acknowledgements}

Authors thank the support of National Natural Science Foundation of China (31771094, 61475164), the National Key Research and Development Program of China (Grant No. 2017YFB1104300 and 2016YFA0200500) and Tianjin Science and Technology Innovation Platform Program (14TXGCCX00017).

\section{Notes and references}

1 D. V. Mcallister, P. M. Wang, S. P. Davis, J. H. Park, P. J. Canatella, M. G. Allen and M. R. Prausnitz, Proc. Natl. Acad. Sci. U. S. A., 2003, 100, 13755-13760.

2 A. Doraiswamy, C. Jin, R. J. Narayan, P. Mageswaran, P. Mente, R. Modi, R. Auyeung, D. B. Chrisey, 
A. Ovsianikov and B. Chichkov, Acta Biomater., 2006, 2, 267275.

3 S. D. Gittard, A. Nguyen, K. Obata, A. Koroleva, R. J. Narayan and B. N. Chichkov, Opt. Express, 2011, 2, 3167-3178.

4 B. Li, H. J. Tan, S. Anastasova, M. Power, F. Seichepine and G. Z. Yang, Biosens. Bioelectron., 2019, 123, 77-84.

5 J. H. Strickler and W. W. Webb, Opt. Lett., 1991, 16, 17801782.

6 H. B. Sun, S. Matsuo and H. Misawa, Appl. Phys. Lett., 1999, 74, 786-788.

7 H. B. Sun, Y. Xu, S. Juodkazis, K. Sun, M. Watanabe, S. Matsuo, H. Misawa and J. Nishii, Opt. Lett., 2001, 26, 325-327.

8 M. Watanabe, S. Juodkazis, H. B. Sun, S. Matsuo and H. Misawa, Appl. Phys. Lett., 2000, 77, 13-15.

9 B. G. Wang, K. Koenig, I. Riemann, R. Krieg and K. J. Halbhuber, Histochem. Cell Biol., 2006, 126, 507-515.

10 D. Wu, S. Z. Wu, L. G. Niu, Q. D. Chen, R. Wang, J. F. Song, H. H. Fang and H. B. Sun, Appl. Phys. Lett., 2010, 97, 031109.

11 C. Vieu, F. Carcenac, A. Pepin, Y. Chen, M. Mejias, A. Lebib, L. Manin-Ferlazzo, L. Couraud and H. Launois, Appl. Surf. Sci., 2000, 164, 111-117.

12 Y. L. Tian, T. J. Isotalo, M. P. Konttinen, J. W. Li, S. Heiskanen, Z. R. Geng and I. J. Maasilta, J. Phys. D: Appl. Phys., 2017, 50, 055302.

13 N. D. Leo, M. Fretto, V. Lacquaniti, C. Cassiago, L. D'Ortenzi, L. Boarino and S. Maggi, IEEE Trans. Appl. Supercond., 2016, 26, 1-5.

14 C. E. Shuck, M. Frazee, A. Gillman, M. T. Beason, I. E. Gunduz, K. Matoua, R. Winarski and A. S. Mukasyan, J. Synchrotron Radiat., 2016, 23, 990-996.

15 T. Billiet, M. Vandenhaute, J. Schelfhout, S. Van Vlierberghe and P. Dubruel, Biomaterials, 2012, 33, 6020-6041.

16 F. P. Melchels, J. Feijen and D. W. Grijpma, Biomaterials, 2010, 31, 6121-6130.

17 S. J. Lee, J. W. Rhie and D. W. Cho, J. Manuf. Sci. Eng., 2008, 130, 021007.

18 P. Gu, X. Zhang, Y. Zeng and B. Ferguson, J. Manuf. Syst., 2001, 20, 250-263.

19 X. Zhang, B. Zhou, Y. Zeng and P. Gu, Robot. Comput. Integr. Manuf., 2002, 18, 41-51.

20 Y. Lin and J. Xu, Adv. Opt. Mater., 2018, 6, 1701359.

21 G. Kumi, C. O. Yanez, K. D. Belfield and J. T. Fourkas, Lab Chip, 2010, 10, 1057-1060.

22 J. F. Xing, M. L. Zheng and X. M. Duan, Chem. Soc. Rev., 2015, 44, 5031-5039.

23 X. B. Yin, N. Fang, X. Zhang, I. B. Martini and B. J. Schwartz, Appl. Phys. Lett., 2002, 81, 3663-3665.

24 W. H. Teh, U. Durig, U. Drechsler, C. G. Smith and H. J. Guntherodt, J. Appl. Phys., 2005, 97, 54907.

25 D. Wu, Q. D. Chen, L. G. Niu, J. N. Wang, J. Wang, R. Wang, H. Xia and H. B. Sun, Lab Chip, 2009, 9, 2391-2394.

26 D. F. Tan, Y. Li, F. J. Qi, H. Yang, Q. H. Gong, X. Z. Dong and X. M. Duan, Appl. Phys. Lett., 2007, 90, 071106.

27 K. Takada, K. Kaneko, Y. D. Li, S. Kawata, Q. D. Chen and H. B. Sun, Appl. Phys. Lett., 2008, 92, 041902.
28 Z. Cai, Y. Liu, C. Zhang, J. Xu, S. Ji, J. Ni, J. Li, Y. Hu, D. Wu and J. Chu, Opt. Lett., 2017, 42, 2483-2486.

29 S. Ji, L. Yang, Y. Hu, J. Ni, W. Du, J. Li, G. Zhao, D. Wu and J. Chu, Small, 2017, 13, 1701190.

30 D. Pan, Z. Cai, S. Ji, S. Fan, P. Wang, Z. Lao, L. Yang, J. Ni, C. Wang, J. Li, Y. Hu, D. Wu, S. Chen and J. Chu, ACS Appl. Mater. Interfaces, 2018, 10, 36369-36376.

31 Z. X. Lao, Y. L. Hu, D. Pan, R. Y. Wang, C. C. Zhang, J. C. Ni, B. Xu, J. W. Li, D. Wu and J. R. Chu, Small, 2017, 13, 1603957. 32 C. Heller, M. Schwentenwein, G. Russmueller, F. Varga, J. Stampfl and R. Liska, J. Polym. Sci., Part A: Polym. Chem., 2009, 47, 6941-6954.

33 X. H. Qin, P. Gruber, M. Markovic, B. Plochberger, E. Klotzsch, J. Stampfl, A. Ovsianikov and R. Liska, Polym. Chem., 2014, 5, 6523-6533.

34 B. Duan, L. A. Hockaday, K. H. Kang and J. T. Butcher, J. Biomed. Mater. Res., 2013, 101, 1255-1264.

35 A. Skardal, J. X. Zhang, L. McCoard, X. Y. Xu, S. Oottamasathien and G. D. Prestwich, Tissue Eng., Part A, 2010, 16, 2675-2685.

36 M. Morra, Biomacromolecules, 2005, 6, 1205-1223.

37 J. B. Baier Leach, K. A. Bivens, C. W. Patrick and C. E. Schmidt, Biotechnol. Bioeng., 2003, 82, 578-589.

38 S. Gerecht, J. A. Burdick, L. S. Ferreira, S. A. Townsend, R. Langer and G. Vunjak-Novakovic, Proc. Natl. Acad. Sci. U. S. A., 2007, 104, 11298-11303.

39 X. Hu, P. Cebe, A. S. Weiss, F. Omenetto and D. L. Kaplan, Mater. Today, 2012, 15, 208-215.

40 Y. L. Sun, Q. Li, S. M. Sun, J. C. Huang, B. Y. Zheng, Q. D. Chen, Z. Z. Shao and H. B. Sun, Nat. Commun., 2015, 6, 8612.

41 A. Ovsianikov, A. Deiwick, S. Van Vlierberghe, P. Dubruel, L. Möller, G. Dräger and B. Chichkov, Biomacromolecules, 2011, 12, 851-858.

42 A. Ovsianikov, A. Deiwick, S. Van Vlierberghe, M. Pflaum, M. Wilhelmi, P. Dubruel and B. Chichkov, Materials, 2011, 4, 288-299.

43 L. Brigo, A. Urciuolo, S. Giulitti, G. Della Giustina, M. Tromayer, R. Liska, N. Elvassore and G. Brusatin, Acta Biomater., 2017, 55, 373-384.

44 S. Engelhardt, E. Hoch, K. Borchers, W. Meyer, H. Kruger, G. E. M Tovar and A. Gillner, Biofabrication, 2011, 3, 025003.

45 E. C. Spivey, E. T. Ritschdorff, J. L. Connell, C. A. McLennon, C. E. Schmidt and J. B. Shear, Adv. Funct. Mater., 2013, 23, 333-339.

46 D. Serien and S. Takeuchi, Appl. Phys. Lett., 2015, 107, 013702.

47 C. L. Lay, Y. H. Lee, M. R. Lee, I. Y. Phang and X. Y. Ling, ACS Appl. Mater. Interfaces, 2016, 8, 8145-8153.

48 X. Gou, M. Zheng, Y. Zhao, X. Dong, F. Jin, J. Xing and X. Duan, Appl. Surf. Sci., 2017, 416, 273-280.

49 S. X. Wei, J. Liu, Y. Y. Zhao, T. B. Zhang, M. L. Zheng, F. Jin, X. Z. Dong, J. F. Xing and X. M. Duan, ACS Appl. Mater. Interfaces, 2017, 9, 42247-42257.

50 J. F. Xing, J. H. Liu, T. B. Zhang, L. Zhang, M. L. Zheng and X. M. Duan, J. Mater. Chem. B, 2014, 2, 4318-4323. 
51 J. F. Xing, X. Z. Dong, W. Q. Chen, X. M. Duan, N. Takeyasu, T. Tanaka and S. Kawata, Appl. Phys. Lett., 2007, 90, 131106.

52 S. Maruo, O. Nakamura and S. Kawata, Opt. Lett., 1997, 22, 132-134.

53 S. Kawata, H. B. Sun, T. Tanaka and K. Takada, Nature, 2001, 412, 697-698.

54 Z. Xiong, X. Z. Dong, W. Q. Chen and X. M. Duan, Appl. Phys. A: Mater. Sci. Process., 2008, 93, 447-452.

55 S. D. Gittard, A. Ovsianikov, N. A. Monteiro-Riviere, J. Lusk, P. Morel, P. Minghetti, C. Lenardi, B. N. Chichkov and R. J. Narayan, J. Diabetes Sci. Technol., 2009, 3, 304-311.

56 Z. Xiong, M. L. Zheng, X. Z. Dong, W. Q. Chen, F. Jin, Z. S. Zhao and X. M. Duan, Soft Matter, 2011, 7, 10353-10359. 57 S. Y. Ji, L. Yang, Y. L. Hu, J. C. Ni, W. Q. Du, J. W. Li, G. Zhao,

D. Wu and J. R. Chu, Small, 2017, 13, 1701190.

58 X. Zhou, Y. Hou and J. Lin, AIP Adv., 2015, 5, 030701.

59 M. Albota, D. Beljonne, J. L. Brédas, J. E. Ehrlich, J. Y. Fu, A. A. Heikal, S. E. Hess, T. Kogej, M. D. Levin and S. R. Marder, Science, 1998, 281, 1653-1656.

60 S. M. Kuebler, S. P. Ananthavel, M. Rumi, S. R. Marder, J. W. Perry, S. Barlow, B. H. Cumpston, D. L. Dyer, J. E. Ehrlich and L. L. Erskine, Nature, 1999, 398, 51-54.

61 J. F. Xing, M. L. Zheng, W. Q. Chen, X. Z. Dong, N. Takeyasu, T. Tanaka, Z. S. Zhao, X. M. Duan and S. Kawata, Phys. Chem. Chem. Phys., 2012, 14, 15785-15792.

62 S. J. Jhaveri, J. D. Mcmullen, R. Sijbesma, L. S. Tan, W. Zipfel and C. K. Ober, Chem. Mater., 2009, 21, 2003-2006.

63 J. F. Xing, L. Liu, X. Y. Song, Y. Y. Zhao, L. Zhang, X. Z. Dong, F. Jin, M. L. Zheng and X. M. Duan, J. Mater. Chem. B, 2015, 3, 8486-8491.

64 Y. C. Zheng, Y. Y. Zhao, M. L. Zheng, S. L. Chen, J. Liu, F. Jin, X. Z. Dong, Z. S. Zhao and X. M. Duan, ACS Appl. Mater. Interfaces, 2019, 11, 1782-1789.

65 S. Nagaraj, S. Easwaramoorthi, J. R. Rao and P. Thanikaivelan, Int. J. Biol. Macromol., 2019, 131, 779-786. 66 S. Basil, L. P. Cunningham, G. D. Pins, K. A. Bush, R. Taboada, A. R. Howell, J. Wang and P. J. Campagnola, Biomacromolecules, 2005, 6, 1465-1474.

67 P. J. Campagnola, D. M. Delguidice, G. A. Epling, K. D. Hoffacker, A. R. Howell, J. D. Pitts and S. L. Goodman, Macromolecules, 2000, 33, 1511-1513.

68 Y. Yağci and I. Reetz, Prog. Polym. Sci., 1998, 23, 1485-1538. 69 M. Aydin, N. Arsu and Y. Yagci, Macromol. Rapid Commun., 2003, 24, 718-723.

70 J. D. Pitts, A. R. Howell, R. Taboada, I. Banerjee, J. Wang, S. L. Goodman and P. J. Campagnola, Photochem. Photobiol., 2002, 76, 135-144.

71 X. Huang, X. P. Wang and Y. X. Zhao, Dyes Pigm., 2017, 141, 413-419.

72 M. Farsari, G. Filippidis, K. Sambani, T. S. Drakakis and C. Fotakis, J. Photochem. Photobiol., A, 2006, 181, 132-135.

73 A. Ovsianikov, M. Gruene, M. Pflaum, L. Koch, F. Maiorana, M. Wilhelmi, A. Haverich and B. Chichkov, Biofabrication, 2010, 2, 014104.

74 T. Weiß, R. Schade, T. Laube, A. Berg, G. Hildebrand, R. Wyrwa, M. Schnabelrauch and K. Liefeith, Adv. Eng. Mater., 2011, 13, B264-B273.
75 E. Scarpa, E. D. Lemma, R. Fiammengo, M. P. Cipolla, F. Pisanello, F. Rizzi and M. De Vittorio, Sens. Actuators, B, 2019, 279, 418-426.

76 A. Ovsianikov, M. Malinauskas, S. Schlie, B. Chichkov, S. Gittard, R. Narayan, M. Lobler, K. Sternberg, K. P. Schmitz and A. Haverich, Acta Biomater., 2011, 7, 967-974.

77 S. D. Gittard, A. Ovsianikov, H. Akar, B. Chichkov, N. A. Monteiro-Riviere, S. Stafslien, B. Chisholm, C. C. Shin, C. M. Shih, S. J. Lin, Y. Y. Su and R. J. Narayan, Adv. Eng. Mater., 2010, 12, B77-B82.

78 C. Lv, X. C. Sun, H. Xia, Y. H. Yu, G. Wang, X. W. Cao, S. X. Li, Y. S. Wang, Q. D. Chen, Y. D. Yu and H. B. Sun, Sens. Actuators, B, 2018, 259, 736-744.

79 A. I. Ciuciu and P. J. Cywiński, $R S C A d v ., 2014$, 4, 4550445516.

80 A. Koroleva, O. Kufelt, S. Schlie-Wolter, U. Hinze and B. Chichkov, Biomed. Tech., 2013, 58, 399-405.

81 O. Kufelt, A. El-Tamer, C. Sehring, S. Schlie-Wolter and B. N. Chichkov, Biomacromolecules, 2014, 15, 650-659.

82 A. Berg, E. T. Oner, J. Combie, B. Schneider, R. Ellinger, J. Weisser, R. Wyrwa and M. Schnabelrauch, Int. J. Biol. Macromol., 2018, 107, 2312-2319.

83 A. Berg, R. Wyrwa, J. Weisser, T. Weiss, R. Schade, G. Hildebrand, K. Liefeith, B. Schneider, R. Ellinger and M. Schnabelrauch, Adv. Eng. Mater., 2011, 13, B274-B284.

84 S. K. Seidlits, C. E. Schmidt and J. B. Shear, Adv. Funct. Mater., 2009, 19, 3543-3551.

85 Y. L. Sun, W. F. Dong, R. Z. Yang, X. Meng, L. Zhang, Q. D. Chen and H. B. Sun, Angew. Chem., Int. Ed. Engl., 2012, 51, 1558-1562.

86 M. H. Tong, N. Huang, W. Zhang, Z. L. Zhou, A. H. W. Ngan, Y. N. Du and B. P. Chan, Sci. Rep., 2016, 6, 20063.

87 K. Kuetemeyer, G. Kensah, M. Heidrich, M. H. Meyer, U. Martin, I. Gruh and A. Heisterkamp, Opt. Express, 2011, 19, 15996-16007.

88 R. Allen, R. Nielson, D. D. Wise and J. B. Shear, Anal. Chem., 2005, 77, 5089-5095.

89 D. S. Hernandez, E. T. Ritschdorff, S. K. Seidlits, C. E. Schmidt and J. B. Shear, J. Mater. Chem. B, 2016, 4, 1818-1826.

90 S. Basu and P. J. Campagnola, J. Biomed. Mater. Res., Part A, 2004, 71, 359-368.

91 J. D. Pitts, P. J. Campagnola, G. A. Epling and S. L. Goodman, Macromolecules, 2000, 33, 1514-1523.

92 Y. L. Sun, D. X. Liu, W. F. Dong, Q. D. Chen and H. B. Sun, Opt. Lett., 2012, 37, 2973-2975.

93 K. C. Cho, C. H. Lien, C. Y. Lin, C. Y. Chang, L. L. H. Huang, P. J. Campagnola, C. Y. Dong and S. J. Chen, Opt. Express, 2011, 19, 11732-11739.

94 M. R. Lee, I. Y. Phang, Y. Cui, Y. H. Lee and X. Y. Ling, Small, 2015, 11, 740-748.

95 B. Kaehr and J. B. Shear, Proc. Natl. Acad. Sci. U. S. A., 2008, 105, 8850-8854.

96 J. Van Hoorick, P. Gruber, M. Markovic, M. Tromayer, J. Van Erps, H. Thienpont, R. Liska, A. Ovsianikov, P. Dubruel and S. Van Vlierberghe, Biomacromolecules, 2017, 18, 3260-3272. 